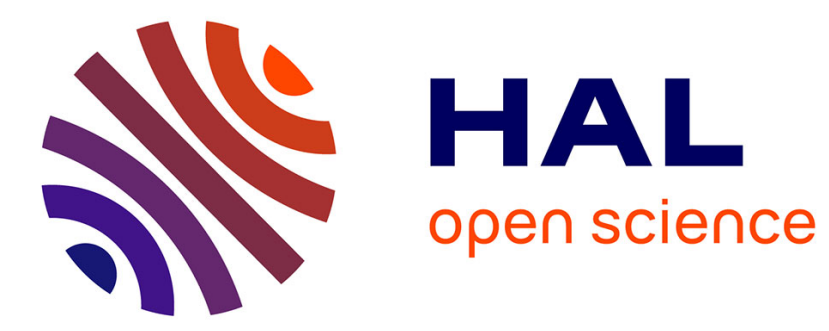

\title{
Track Initiation of Low-Earth-Orbit Objects using Statistical Modeling of Sparse Observations
}

Thibaut Castaings, Benjamin Pannetier, Florent Muller, Michèle Rombaut

\section{To cite this version:}

Thibaut Castaings, Benjamin Pannetier, Florent Muller, Michèle Rombaut. Track Initiation of Low-Earth-Orbit Objects using Statistical Modeling of Sparse Observations. IEEE Transactions on Aerospace and Electronic Systems, 2014, 51 (1), pp.258-269. 10.1109/TAES.2014.130484. hal01070959

\section{HAL Id: hal-01070959 \\ https://hal.science/hal-01070959}

Submitted on 2 Oct 2014

HAL is a multi-disciplinary open access archive for the deposit and dissemination of scientific research documents, whether they are published or not. The documents may come from teaching and research institutions in France or abroad, or from public or private research centers.
L'archive ouverte pluridisciplinaire HAL, est destinée au dépôt et à la diffusion de documents scientifiques de niveau recherche, publiés ou non, émanant des établissements d'enseignement et de recherche français ou étrangers, des laboratoires publics ou privés. 


\title{
Track Initiation of Low-Earth-Orbit Objects using Statistical Modeling of Sparse Observations
}

\author{
Thibaut Castaings ${ }^{1,2}$, Benjamin Pannetier ${ }^{1}$, Florent Muller ${ }^{1}$, and Michèle Rombaut ${ }^{2}$ \\ ${ }^{1}$ ONERA, Chemin de la Hunière, 91123 Palaiseau, France \\ ${ }^{2}$ GIPSA-lab, 11 rue des Mathématiques, 38402 Saint Martin d'Hères, France \\ castaings.t@gmail.com, \{benjamin.pannetier, florent.muller\}@onera.fr, \\ michele.rombaut@gipsa-lab.grenoble-inp.fr
}

\begin{abstract}
In this paper, we investigate a new track initiation technique enabling the use of a low-cost radar system for Low-Earth-Orbit surveillance. This technique is based on a first association of observations with little ambiguity followed by a fast Initial Orbit Determination. This study supports the feasibility of the system as this technique shows a coverage of $84,4 \%$ within 6 days, with a combinatorial complexity kept under control when assessed in a realistic multitarget tracking context.
\end{abstract}

Index Terms-Multiple hypothesis tracking, track initiation, Low-Earth-Orbit, space debris, narrow fence, short arcs, orbit determination, hypothesis testing.

\section{INTRODUCTION}

Following recent events, tracking the small space debris for collision avoidance has become a topic of great interest [1], [2], [3], [4]. The risk of collision in Mid-EarthOrbit (MEO) and Geosynchronous-Earth-Orbit (GEO) is of lesser concern to operators, as compared to the risk of collision in Low-Earth-Orbit (LEO) which is much higher due to a high debris density at this range of altitudes. Consequently, this study focuses only on LEO objects (those having a geodetic altitude between $160 \mathrm{Km}$ and $2000 \mathrm{Km}$, i.e. the revolution period ranges between 88 minutes and 127 minutes). For the LEO objects, radarbased sensors [5], [6] are more adapted than optical sensors for illumination reasons. Those sensors use a large Field Of Regard (FOR), making the estimation of an orbit at each pass of an object in the FOR possible. The association of several passes can be decided from the correlation of their orbits in order to keep the combinatorial complexity under control during the tracking. Such systems being part of the United States Space Surveillance Network, the
United States Space Command (USSPACECOM) is able to provide the Space-Track Two-Lines Element (TLEs) catalog [7] containing the orbits of more than 15,000 objects with a diameter greater than $10 \mathrm{~cm}$, including the maneuvering satellites. However, the estimated number of smaller lethal objects is even greater [8], [9] and has become a significant threat for space systems.

In order to track ever smaller and more numerous objects, current sensors and methods are pushed to their limits. The best trade-off criterion to detect smaller objects with a survey radar is to increase the frequency of the emitted radio-wave and to reduce the FOR. On the one hand, a higher frequency leads to a higher radar crosssection for objects of smaller size, because a resonance occurs when the wavelength is about the size of the scatterer (Mie scattering) [10], [11]. On the other hand, the wavelength appears in the denominator of the Signalto-Noise Ratio (SNR), making it decrease as the frequency increases. To counteract this unfavorable effect, a reduced FOR, for instance a FOR of a wide cross-elevation and narrow elevation (narrow detection fence), is a possible option. As a result, a proper increase in frequency and a proper reduction of the FOR yield an increased SNR for smaller objects.

Although this option is less costly, it involves less observations (for instance one or two observations per pass), i.e. Short Arcs (SAs), from which an orbit cannot be estimated using the existing techniques, making even harder the problem of associating the observations originated from the numerous LEO objects and filtering the generally high number of False Alarms (FAs). Currently, no reliable data processing approach has been proposed to address this 
problem. This study aims at developing a method enabling to process SAs in order to support the feasibility, from a data processing perspective, of a system using a reduced FOR for cataloging the small LEO objects.

In this paper, we propose and investigate a new track initiation technique enabling first the association of SAs at one revolution of interval with little ambiguity and then a fast Initial Orbit Determination (IOD). This technique follows from two preliminary studies that we have presented in the conference papers [12] and [13]. The association of observations at one revolution of interval relies on the characterization of correct pairs of SAs in a wellchosen feature space, and the principle of the proposed IOD lies in a temporary circular orbit approximation. The combinatorial complexity being the main difficulty encountered, a Track-Oriented Multiple Hypothesis Tracker (TOMHT) [14], [15], [16], [17], [18] has been adapted and implemented to assess the efficiency of the proposed track initiation technique in a realistic simulation.

Figure (1) shows examples of simulated observations (in a local spherical frame of reference East-North-Up (ENU)) obtained over a period of about 9 hours with: a radar of large Field Of Regard $\left(160^{\circ} \times 20^{\circ}\right)$ (top); a radar of narrow FOR $\left(160^{\circ} \times 2^{\circ}\right)$ (bottom).

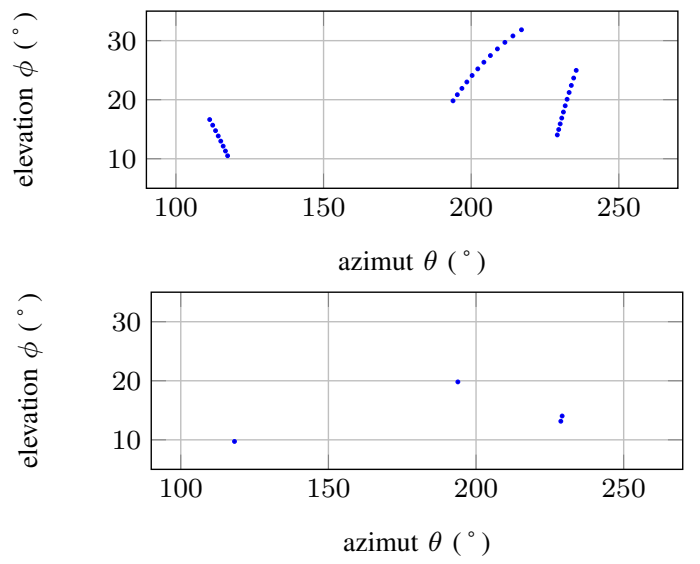

Figure 1. Example of simulated observations of the satellite "THOR ABLESTAR DEB" from the Space-Track TLE catalog that would be accumulated over a period of about 9 hours in a topocentric spherical frame of reference ENU using: a radar of large Field Of Regard $\left(160^{\circ} \times 20^{\circ}\right)$ (top); a radar of narrow FOR $\left(160^{\circ} \times 2^{\circ}\right)$ (bottom). In both cases the sensor is located at $45^{\circ}$ latitude with a Line Of Sight (LOS) of $20^{\circ}$ of elevation. No False Alarm (FA) has been added nor any observation noise. Range is not represented.

In the large-FOR case, observations can be associated into arcs using well-known data association techniques such as, e.g. a Global Nearest Neighbor approach [14], [19]. Then, an Initial Orbit (IO) is estimated with a sufficient precision for each arc using Initial Orbit Determination (IOD) techniques such as Gibbs method, Herrick-Gibbs method, Lambert's or even Battin's method depending on the available data [20], [21]. The orbits are then propagated to relevant times in order to assess a possible correlation [22], [23]. When a sufficient number of observations are associated, a least-square minimization is performed in order to get a Definitive Orbit (DO), i.e. an orbit of high accuracy [22], [24].

In the narrow-FOR case, the information contained in each arc is highly degraded: only one or two observations with a high redundancy - i.e. a Short Arc (SA) - are available for each pass of an object.

An orbit cannot be estimated from SAs using existing techniques because they require a minimal number of observations with a small amount of redundancy (i.e. the observations should be spread over an arc of sufficient length with regard to the measurement noise in order to meet requirements of coplanarity). In a realistic scenario, the SAs must therefore be associated at one or several revolutions of interval before an IO calculation is attempted whereas the high number of objects and FAs implies a tremendous number of associative hypotheses for each new detection (one revolution takes 88 to 127 minutes).
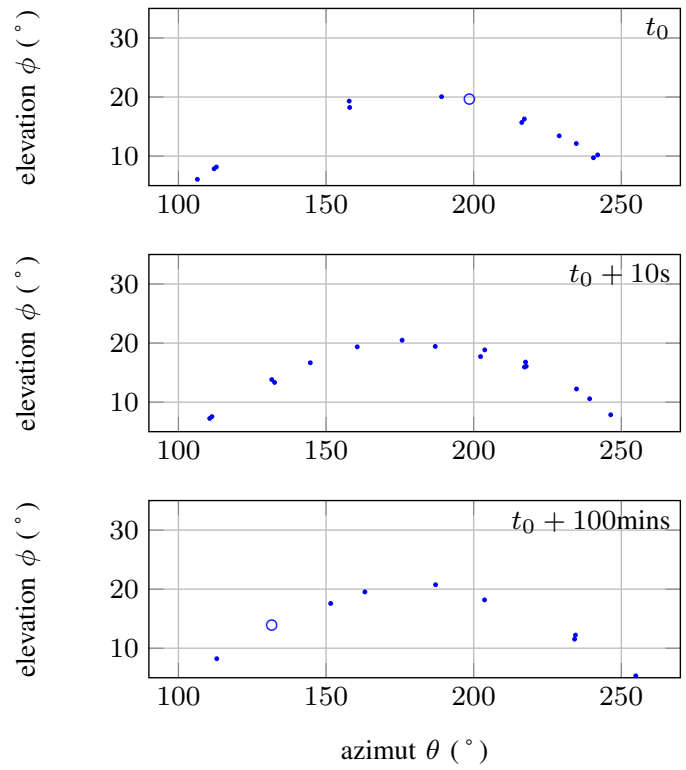

Figure 2. Example of simulated observations of the objects contained in the Space-Track catalog obtained with a radar of narrow FOR $\left(160^{\circ} \times 2^{\circ}\right)$ located at $45^{\circ}$ latitude with a Line Of Sight (LOS) of $20^{\circ}$ of elevation, in a topocentric spherical frame of reference ENU. The circles represent observations of one particular object. No False Alarm (FA) has been added. Range is not represented. 
Figure 2 shows a couple of scans resulting from simulated detections of the Space-Track TLE catalog objects that would be obtained with a radar of narrow Field Of Regard $\left(160^{\circ} \times 2^{\circ}\right)$. The observations of an object generally occur in one or several revolutions of interval and should be associated within a prohibitive number of possibilities. The approach proposed and investigated in this paper addresses this problem.

This paper is organized as follows: we first briefly state the hypotheses related to the sensor and the dynamic model in Section II. The principle of the new tracking block is then presented in Section III. Techniques to associate the observations from scan to scan as well as the observations from revolution to revolution are explained first. Then, the calculus of a circular orbit enabling the use of more regular techniques for the following observation-to-track associations (and thus completing the track initiation) is described. Choices in the implementation of the TOMHT are discussed in Section IV and its performance is assessed and discussed in Section V. Finally, a conclusion is drawn in Section VI.

\section{SPACE OBJECT TRACKING}

Tracking an object consists in estimating its position in time from known (observed) past positions. This requires the use of a sensor and a dynamic model. In this section, the sensor characteristics are presented. The dynamic model (namely the Simplified General Perturbation model, or SGP) used in this study is also briefly presented although the details of its equations are not recalled for the sake of simplicity (for that, the reader should refer to the reference papers [7], [24]). Finally, the principle of the well-known Gauss-Newton Least-Square algorithm used in this study for the Definitive Orbit (DO) estimation is briefly recalled.

\section{A. Sensor Characteristics}

Under certain technological conditions, some basic electromagnetic considerations [25] lead to the conclusion that the best trade-off criterion to detect smaller objects is to increase the frequency of the emitted radio-wave [10], which requires a reduced Field Of Regard (FOR) to moderate a possible cost increase of the sensor. Typical specifications for the design of actual space surveillance systems are adopted in the simulations:

- The sensor is located at latitude $45^{\circ}$ with a FOR oriented toward the equator with arbitrarily-chosen $20^{\circ}$ Line-Of-Sight (LOS) elevation. The FOR has a wide cross-elevation $\left(160^{\circ}\right)$ and a narrow elevation $\left(2^{\circ}\right)$,
- The sensor produces one scan of the whole FOR every 10s (Track-While-Scan mode),

- The sensor provides range $\rho$, azimuth $\theta$ and elevation $\phi$ measurements (pulsed mono-static) for each detection, with a precision of $\sigma_{\rho}=30 \mathrm{~m}$ in range, $\sigma_{\theta}=0.2^{\circ}$ in cross-elevation and $\sigma_{\phi}=0.2^{\circ}$ in elevation,

- The false alarm rate is $1 \mathrm{FA} / \mathrm{s}$.

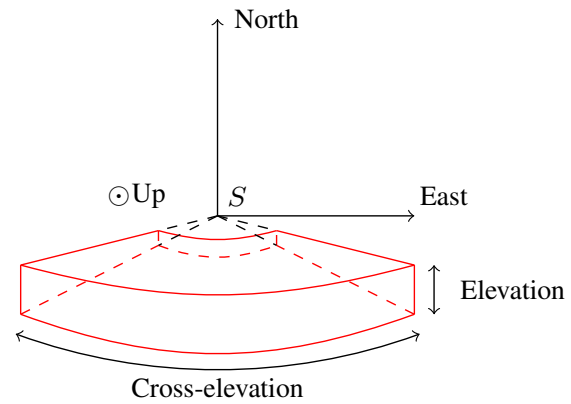

Figure 3. Shape of the Field Of Regard (FOR) in an East-North-Up frame of reference. The FOR is represented in red.

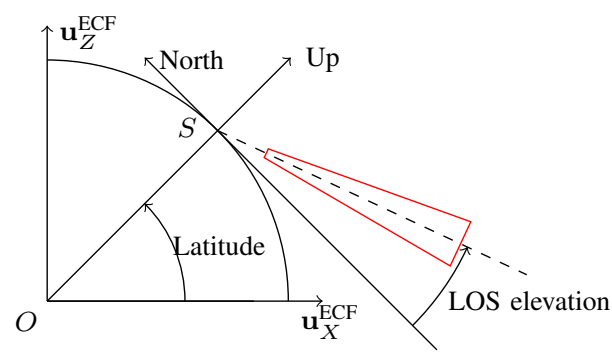

Figure 4. Orientation of the Field Of Regard (FOR) in a geocentric frame of reference.

Figure 3 and 4 represent the FOR shape and orientation. The sensor located at position $S$ provides 3D position measurements $\{\rho, \theta, \phi\}$ in a topocentric, spherical frame of reference East-North-Up (ENU). Those measurements can be expressed as well in an Earth-Centered Inertial (ECI) frame of reference $\left\{O, \mathbf{u}_{X}^{\mathrm{ECI}}, \mathbf{u}_{Y}^{\mathrm{ECI}}, \mathbf{u}_{Z}^{\mathrm{ECI}}\right\}$ or in an Earth-Centered Fixed (ECF) frame of reference $\left\{O, \mathbf{u}_{X}^{\mathrm{ECF}}, \mathbf{u}_{Y}^{\mathrm{ECF}}, \mathbf{u}_{Z}^{\mathrm{ECF}}\right\}$ (an ECF follows the rotation of the Earth in an ECI).

The sensor as defined here provides observations of range, azimuth and elevation grouped into scans so that:

$$
\mathbf{z}_{j}(k)=\left\{\rho_{j}(k), \theta_{j}(k), \phi_{j}(k)\right\}
$$

is the $j$-th observation of the $k$-th scan degraded with a Gaussian noise of covariance $\mathbf{R}=\operatorname{diag}\left(\sigma_{\rho}^{2}, \sigma_{\theta}^{2}, \sigma_{\phi}^{2}\right)$, 


$$
Z(k)=\left\{\mathbf{z}_{j}(k)\right\}_{j \in\left[1, \ldots, m_{k}\right]}
$$

is the set of $m_{k}$ observations constituting the $k$-th scan.

\section{B. The Simplified General Perturbation model}

Predicting the position of an object taken from the Space-Track TLE catalog requires the use of a Simple General Perturbation (SGP) model. This model has been developed and described in [7] and predicts the effect of perturbations caused by the Earth's five first spherical harmonics and the atmospheric drag [7], [24], [26]. The chosen orbital state components are given in Table I.

\section{Table I}

DEFINITIONS OF THE CHOSEN ORBITAL STATE COMPONENTS.

$t \quad$ : Element Set Epoch,

$n \quad:$ Mean Motion (revolutions/day), related to Semi-major axis $a$ by Kepler's third law

$e \quad:$ Eccentricity

$i \quad$ : Orbit Inclination (degrees)

$\Omega \quad$ : Right Ascension of Ascending Node (degrees)

$\omega \quad$ : Argument of Perigee (degrees)

$M \quad$ : Mean Anomaly (degrees)

$B * \quad$ : Drag Term

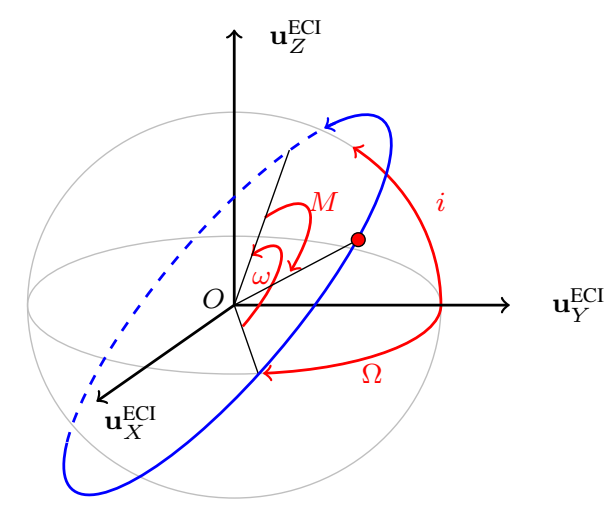

Figure 5. Example of an orbit represented by an ellipse in an ECI frame of reference.

For each object, the state vector $\mathbf{X}_{t}$ (all the components vary with time) is associated to an orbit and defined as $[n, e, i, \Omega, \omega, M, B *]^{\mathrm{T}}$ or $[n, e, i, \Omega, \omega, M]^{\mathrm{T}}$ depending on the availability of $B *$ (the drag term $B *$ has an influence on a long time span and might be unavailable for short tracks), and $\mathbf{P}_{\mathbf{X X}}$ its covariance matrix.

The published versions [7], [24] of the SGP propagation model provide a function $f_{\mathrm{SGP}}$ defined so that:

$$
f_{\mathrm{SGP}}\left(\mathbf{X}_{t_{0}}, t\right)=(\mathbf{r}, \mathbf{v})_{\mathbf{X}_{t_{0}}, t}
$$

where $\mathbf{X}_{t_{0}}$ is the orbital state at time $t_{0}$ and $(\mathbf{r}, \mathbf{v}) \mathbf{X}_{t_{0}}, t$ is the couple position-velocity associated to $\mathbf{X}_{t_{0}}$ at time $t$ in an ECF frame of reference. The function $f_{\mathrm{SGP}}$ enables therefore the propagation of $\mathbf{X}_{t_{0}}$ to time $t$ and deduces position $\mathbf{r}$ and velocity $\mathbf{v}$ from a propagated orbit $\overline{\mathbf{X}}_{t}$.

Then, an observation $\overline{\mathbf{z}}_{\mathbf{X}_{t_{0}}, t}$ can be predicted by selecting the position $\mathbf{r}$ and expressing it in an ENU frame of reference through some mapping function $h$.

\section{Definitive orbit estimation}

A state vector $\mathbf{X}_{t}$ and its covariance matrix $\mathbf{P}_{\mathbf{X X}, t}$ are estimated from a set of $m$ observations $\left\{\mathbf{z}_{t_{i}}\right\}_{i=1, \ldots, m}$ and the SGP model in the case of space object tracking, using for example a least square minimization criterion which can be written as:

$$
\mathbf{X}_{t}=\underset{\mathbf{X}}{\operatorname{argmin}} \sum_{i=1}^{m}\left(\overline{\mathbf{z}}_{\mathbf{X}, t_{i}}-\mathbf{z}_{t_{i}}\right)^{\mathrm{T}} \mathbf{R}^{-1}\left(\overline{\mathbf{z}}_{\mathbf{X}, t_{i}}-\mathbf{z}_{t_{i}}\right)
$$

where $\overline{\mathbf{z}}_{\mathbf{X}, t_{i}}$ and $\mathbf{z}_{t_{i}}$ refer to, respectively, the $i$-th predicted observation and the $i$-th observation. In this study, the Gauss-Newton Least-Square (GNLS) algorithm [27] is used to compute Definitive Orbits (DO). Estimating a definitive orbit requires associated observations and a good initial guess (i.e. close enough to the solution) [22]. The use of a least-square criterion to estimate a DO assumes observability, i.e. the solution is unique. In the case of non-observability, i.e. in a case where the number of non-redundant observations is too low with respect to the measurement noise and the complexity of the underlying model, the algorithm can diverge or converge to an incorrect value.

\section{TRACK INITIATION}

This study is focused on the track initiation (i.e. the association process that enables a DO estimation) of a satellite in LEO in a sparse data context such as using a narrow fence-type radar. An object produces one or two observations at each pass in a narrow FOR with a large amount of redundancy, disabling the estimation of an orbit at each pass. To this day, to the best of our knowledge, no approach has been proposed to address this issue. In this paper, we therefore propose new techniques to first associate observations at one revolution of interval and then to compute an Initial Orbit (IO) at a low computational cost. In this section, the principles of these methods are described. 


\section{A. Association of observations in successive scans}

Associating observations in successive scans into SAs (arcs of one or two observations per pass) makes it possible to reduce the number of redundant tracks and can be done using elementary techniques. The assumption is that two observations $\mathbf{z}_{i}(k)$ and $\mathbf{z}_{j}(k+1)$ are close in an ECI frame of reference. SAs are formed by comparing some distances $d_{r}$ and $d_{r \lambda}$ between the observations $\mathbf{z}_{i}(k)$ and $\mathbf{z}_{j}(k+1)$ in successive scans to some thresholds $\gamma_{r}$ and $\gamma_{r \lambda}$. These distances are defined in an ECI frame of reference: $d_{r}$ is their difference of radius and $d_{r \lambda}$ is the length of the arc of longitude between them at altitude of $\mathbf{z}_{i}$ (see Figure 6). To associate two observations $\mathbf{z}_{i}(k)$ and $\mathbf{z}_{j}(k+1)$, the distances must be less than the thresholds $\gamma_{r}$ and $\gamma_{r \lambda}$ which are chosen so that more than $95 \%$ of a test sample consisting in simulated pairs of observations originated from the same object in successive scans fall below them, this threshold value resulting from a trade-off between recall and accuracy.

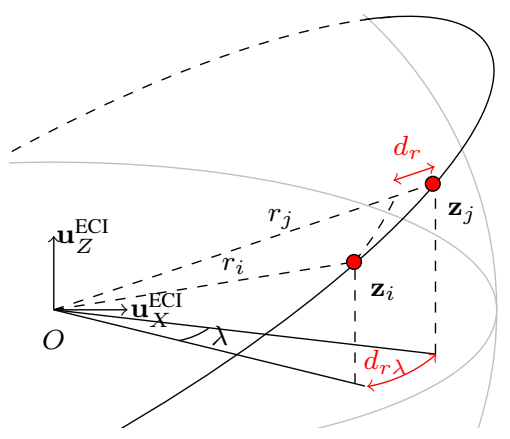

Figure 6. Example of $d_{r}$ and $d_{r \lambda}$ in an ECI frame of reference. $\lambda$ is the difference of the longitudes of the observations in an ECI frame of reference.

\section{B. Association of observations at one revolution of interval}

Associating at least two Short Arcs (SAs) into a track is necessary because only one SA is not sufficient to estimate an orbit. To this purpose, this method relies on the high identifiability of pairs of observations that originated from the same object at one revolution of interval: generally speaking, a non-maneuvering satellite takes "neighboring" positions from one revolution to the other in an ECI frame of reference. Simulation results show that more than $99 \%$ of the LEO objects of the Space-Track TLE catalog appear in the FOR at one revolution of interval at least once over a 30-days time span in the sensor setup described in Section II. In [12], we show that the association of observations originated from the same object at one revolution of interval can be achieved with a low ambiguity by extracting features from every pair of SAs and comparing them to a predefined pattern.

1) Feature extraction: A neighborhood of two observations at one revolution of interval in an ECI frame of reference can be defined as the Euclidean distance between them. However in the case of the LEO objects and a narrow fence-type radar, the Euclidean distance is pessimistic because its symmetry neglects the information lying in the shape of the FOR or in the specificities of the LEO population. As a consequence, we propose to extract the following feature vector $\mathbf{x}_{i, j} \in \mathbb{R}^{5}$ from a pair of observations $\mathbf{z}_{i}$ and $\mathbf{z}_{j}$ at one revolution of interval:

$$
\mathbf{x}_{i, j}=\left(\begin{array}{c}
\tau\left(\mathbf{z}_{i}, \mathbf{z}_{j}\right) \\
d_{r}\left(\mathbf{z}_{i}, \mathbf{z}_{j}\right) \\
d_{r \lambda}\left(\mathbf{z}_{i}, \mathbf{z}_{j}\right) \\
d_{\theta}\left(\mathbf{z}_{i}, \mathbf{z}_{j}\right) \\
r\left(\mathbf{z}_{i}\right)
\end{array}\right)
$$

where the following notations are used. In an ECI frame of reference:

$\tau\left(\mathbf{z}_{i}, \mathbf{z}_{j}\right)$ is the time difference between $\mathbf{z}_{i}$ and $\mathbf{z}_{j}$, $d_{r}\left(\mathbf{z}_{i}, \mathbf{z}_{j}\right)$ is their difference of radius $r$,

$d_{r \lambda}\left(\mathbf{z}_{i}, \mathbf{z}_{j}\right)$ is the length of the arc of longitude between $\mathbf{z}_{i}$ and $\mathbf{z}_{j}$ at the altitude $r\left(\mathbf{z}_{i}\right)$ of $\mathbf{z}_{i}$,

$d_{\theta}\left(\mathbf{z}_{i}, \mathbf{z}_{j}\right)$ is the difference of azimuth of detection $\theta$.

The feature $\tau$ depends on $r\left(\mathbf{z}_{i}\right)$ according to Kepler's third law, assuming a circular orbit. As for $d_{r}$ and $d_{r \lambda}$, they are correlated with $d_{\theta}$. The use of $\tau, d_{r}, d_{r \lambda}$ and $r$ as features showed good results in simulation [12]. In this study, the use of $d_{\theta}$ enables a better precision through its influence on $d_{r}$ and $d_{r \lambda}$.

When the correlation between two SAs containing several observations has to be tested, mean values are used.

2) Pattern identification: We aim at discriminating the pairs of observations originated from the same object (hypothesis $H_{1}$ ) at one revolution of interval from the pairs of observations originated from different objects or FAs (hypothesis $H_{0}$ ). To this purpose, we simulate the detections of the LEO objects of the Space-Track TLE catalog in the sensor set-up defined in the prequel, associate the observations using ground-truth data from the catalog and extract a set of feature vectors $\left\{\mathbf{x}_{H_{1}}\right\}$ from the observations at one revolution of interval. Then, a boundary encompassing most of the feature vectors $\mathbf{x}_{H_{1}}$ is defined to enable the testing of candidate pairs of observations in an online procedure: we estimate the $(1-\nu)$-quantile $Q(1-\nu)$ defined as the minimal volume $C$ on $\mathbb{R}^{5}$ that encompasses at least a fraction $1-\nu$ of the underlying probability distribution of the set $\left\{\mathbf{x}_{H_{1}}\right\}$, 
measured by $p$, as shown in Eq. (6) where $\mathbb{B}\left(\mathbb{R}^{5}\right)$ is a class of measurable subsets of $\mathbb{R}^{5}$ and $\mu$ is a Lebesgue measure on this set.

$$
Q(1-\nu)=\underset{C \in \mathbb{B}\left(\mathbb{R}^{5}\right), p(C)>1-\nu}{\arg \min } \mu(C)
$$

We solved Eq. (6) by a One-Class Support Vector Machine (One-Class SVM) [28]. This extension of the better-known bi-class SVM [29][30][31] requires one set of "labeled data" $\left\{\mathbf{x}_{H_{1}}\right\}$ (i.e. a set of features corresponding to the class of vectors to be recognized) as input during training while being able to deal with unlabeled data $\{\mathbf{x}\}$ in the online procedure. More details are given in Appendix.

3) Hypothesis test: The number of valid pairs can be further reduced using a likelihood ratio $L_{i, j}$, testing the hypothesis $H_{1}$ "All the observations of the track originated from the same object" against the hypothesis $H_{0}$ "All the observations of the track originated from different objects or FAs" :

$$
L_{i, j}=\log \frac{p\left(\mathbf{x}_{i, j} \mid H_{1}\right)}{p\left(\mathbf{x}_{i, j} \mid H_{0}\right)}
$$

where $p\left(\mathbf{x}_{i, j} \mid H_{1}\right)$ can be read directly from an estimation of the distribution inside the boundary (in this study, the Parzen-Rosenblatt windows [32] are used) and $p\left(\mathbf{x}_{i, j} \mid H_{0}\right)$ is defined according to [33] as the density of extraneous observations:

$$
p\left(\mathbf{x}_{i, j} \mid H_{0}\right)=\frac{N}{\mu(C)}
$$

where $N$ is the number of observations $\mathbf{z}_{j}$ forming a valid pair with $\mathbf{z}_{i}$ and $\mu(C)$ the volume of $C$ resulting from solving Eq. (6). The likelihood ratio $L_{i, j}$ is compared to a threshold defined according to the standard Sequential Probability Ratio Test (SPRT) [34] in order to eliminate further candidate pairs of observations.

4) Preliminary results: In this study, the pattern presented herein before has been computed using the observations resulting from the simulation of the LEO objects of the Space-Track TLE catalog and the sensor set-up defined in Section II, over a 30-days timespan. A One-Class SVM has been used on the extracted pairs of observations with $\nu=0.05$. The output pattern enables the correlation of 7.45 pairs of SAs for each new SA on the mean, the cases where no valid pair could be found being excluded. The associated recall rate is $90.89 \%$ over a set of more than 26,000 SAs.

\section{Orbit determination from a pair of observations at one revolution of interval}

To initiate the tracking, a state $\mathbf{X}$ (the orbital parameters) should be computed for each pair of correlated observations at one revolution of interval resulting from the association technique presented in the prequel. Initial Orbit Determination (IOD) has been widely studied and many approaches and extensions have been developed [20]. When two observations are available, Lambert's problem can be solved even for multiple revolutions [21]. However, this processing is computationally intense especially when the observation noise is taken into account. This is critical in our application since the number of hypotheses to test is very high. Moreover, the iterative processes needed in the resolution of Lambert's problem may diverge due to the closeness of the two positions in an ECI frame of reference with regard to the observation noise. This is related to the low state observability also encountered in other studies dealing with IOD using sparse data such as [35]. In [13], we showed that a temporary circular orbit could be taken as an Initial Orbit in order to find a third SA and process a definitive orbit determination for most of the LEO satellites contained in the Space-Track TLE catalog.

1) Determination of an initial circular orbit: A circular orbit is defined by a null eccentricity $(e=0)$. In this case, the parameter $\omega$ is meaningless and can be set to a null value as well. Then, let $\mathbf{X}_{4}$ refer to the state vector $[n, i, \Omega$, $M]^{T}$. The parameters $i, \Omega$ and $M$ can then be computed easily with simple geometrical considerations and $n$ is known within a good accuracy because the observations are at about one revolution of interval. The formulas are straightforward for two observations $\mathbf{z}_{1}$ and $\mathbf{z}_{2}$ :

$$
\begin{aligned}
n & \simeq \frac{2 \pi \pm \delta}{\tau} \\
i & \simeq \arccos \frac{\mathbf{p} \cdot \mathbf{u}_{Z}}{\|\mathbf{p}\|} \\
\Omega & \simeq \frac{\pi}{2} \pm \arccos \frac{\mathbf{p} \cdot \mathbf{u}_{X}}{\left\|\mathbf{p} \cdot \mathbf{u}_{X}+\mathbf{p} \cdot \mathbf{u}_{Y}\right\|} \\
M & \simeq \pm \arccos \frac{\cos \Omega \mathbf{r}_{1} \cdot \mathbf{u}_{X}+\sin \Omega \mathbf{r}_{1} \cdot \mathbf{u}_{Y}}{\left\|\mathbf{r}_{1}\right\|}
\end{aligned}
$$

where $\mathbf{r}_{1}$ and $\mathbf{r}_{2}$ are the position vectors associated to $\mathbf{z}_{1}$ and $\mathbf{z}_{2}$ in a Euclidean ECI frame of reference with unit vectors $\mathbf{u}_{X}, \mathbf{u}_{Y}$ and $\mathbf{u}_{Z}$,

$\delta$ is the angle formed by $\mathbf{r}_{1}$ and $\mathbf{r}_{2}$,

$\tau$ is the time difference between $\mathbf{z}_{1}$ and $\mathbf{z}_{2}$,

$\mathbf{p}$ is the cross product of $\mathbf{r}_{1}$ and $\mathbf{r}_{2}$.

In order to take into account the observation noise (i.e. to compute a covariance matrix associated with an estimated orbit) a Monte Carlo method is used. Random 
samples are drawn from the noise distribution (Gaussian noise of covariance $\left.\operatorname{diag}\left(\sigma_{\rho}^{2}, \sigma_{\theta}^{2}, \sigma_{\phi}^{2}\right)\right)$ and used to generate samples of a distribution of circular orbits. From these, the mean and covariance are computed and taken as an initial circular orbit $\left\{\mathbf{X}_{4}, \mathbf{P}_{\mathbf{X X}, 4}\right\}$ to initiate the tracking. A diagonal matrix $\mathbf{D}_{\mathbf{X X}, 4}=\operatorname{diag}\left(\kappa_{1}, \kappa_{2}, \kappa_{3}, \kappa_{4}\right)$ is added to $\mathbf{P}_{\mathbf{X X}, 4}$ in order to modelize the impact of the circular orbit approximation.

2) Association of a third observation: A circular orbit has been estimated from two observations at one revolution of interval. A third SA at several revolutions of interval must be associated to enable the estimation of a Definitive Orbit (DO) and initiate the tracking.

In this study, the Unscented Transform (UT) [36] is chosen for its performance and low computational cost. The principle of the UT lies in the computation and propagation of specific points called "sigma-points" which have the same statistical features as the distribution defined by $\left\{\mathbf{X}, \mathbf{P}_{\mathbf{X X}}\right\}$. The sigma-points propagated through $f_{\mathrm{SGP}}$ are used to estimate the distribution of the predicted observation $\left\{\overline{\mathbf{z}}, \mathbf{P}_{\overline{\mathbf{z}} \overline{\mathbf{z}}}\right\}$, where $\mathbf{P}_{\overline{\mathbf{z}} \overline{\mathbf{z}}}$ is the covariance matrix of $\overline{\mathbf{z}}$ (see Appendix for more details). A validation gate is obtained from such a distribution as the domain where the cumulative density function of $\left\{\overline{\mathbf{z}}, \mathbf{P}_{\overline{\mathbf{z}} \mathbf{z}}\right\}$ is smaller than a predefined threshold $\gamma$. Usually and in this study, $\gamma$ is set to $95 \%$.

The association of a third observation (it may appear several hours later) enables the testing of the convergence of a Gauss-Newton Least-Square (GNLS) algorithm for a reduced number of candidate observations. If the GNLS algorithm converges, then it provides the sequence of observations with a definitive orbital state $\left\{\mathbf{X}, \mathbf{P}_{\mathbf{X X}}\right\}$.

In [13], simulations showed that in our case, 17.71 SAs on the mean are correlated with a two-SA track using an initial circular orbit before an observation enables the convergence of a GNLS algorithm.

\section{Track-Oriented Multiple Hypothesis TRACKING (TOMHT)}

The approach presented in the prequel generates many possible tracks, including redundant tracks corresponding to the same object or tracks that do not correspond to any object. The TOMHT algorithm aims at associating observations into tracks representing actual targets and to estimate a state for each detected target. The TOMHT has been first proposed in [15] and further modified by other authors, such as [14], [16], [17].

\section{A. Principle}

Initially, tracks are formed from new observations and existing tracks using for instance an UT regardless to whether two tracks share an observation, resulting in a high number of association hypotheses (tracks). Then, the low quality tracks are deleted and the surviving tracks are combined into feasible hypotheses (hypotheses where tracks do not share any observation). After a hypothesislevel track deletion step, the remaining tracks are updated, merged and propagated to the time of the next scan.

1) Track-level pruning: To prevent the number of hypotheses from exploding, the low quality tracks are deleted. For that, a score is defined for each track and compared to thresholds to delete or confirm the track accordingly. Usually, the log-likelihood ratio $L^{k, l}$, where $l$ is a track index and $k$ denotes the scan number, is chosen and expressed as Eq. (13), where $H_{1}$ is the hypothesis "All the observations of the track originated from the same object", $H_{0}$ is the hypothesis "All the observations of the track originated from different objects or FAs" and $c$ is a start value reflecting the probabilities of $H_{1}$ and $H_{0}$, i.e. $c=\log \frac{P\left(H_{1}\right)}{P\left(H_{0}\right)}$.

$$
L^{k, l}=\log \frac{p\left(T^{k, l} \mid H_{1}\right)}{p\left(T^{k, l} \mid H_{0}\right)}+c
$$

$L^{k, l}$ is computed recursively as new observations are associated to the track. The quantity $p\left(T^{k, l} \mid H_{1}\right)$ is computed using Mahalanobis distances between predicted observations and the new observations. As for $p\left(T^{k, l} \mid H_{0}\right)$, it uses the density of extraneous measurements as in [14], [33]. The threshold values for track deletion and track confirmation can be defined according to the standard Sequential Probability Ratio Test (SPRT) formulation [34]. The SPRT involves $\alpha$, the error of type I, and $\beta$, the error of type II in the calculation of the thresholds.

2) Hypothesis-level pruning: After a track-level deletion step using the SPRT, the surviving tracks are separated into clusters (sets of tracks bound by common observations) so that the association problem is divided into independent smaller problems.

For each cluster, the tracks are combined into feasible hypotheses, i.e. into subsets such that two tracks cannot share directly any observation. In order to delete tracks with regard to global considerations, the "hypothesis-level" probability of each track in each cluster is computed from the likelihood of each track the cluster contains [14] so the tracks of insufficient probability may be deleted. This hypothesis-level pruning strategy is not well suited to the track initiation technique proposed in this study because it yields categorical probabilities (the dynamic model is very 
deterministic when observable). It is therefore not used in the simulations.

$N$-scan pruning is another popular pruning approach. It consists in the deletion of all the association hypotheses that do not include the $N$-th newest observation of the most likely track for each target. This method has shown to be effective in many applications and has been implemented in this study. In the simulations, setting $N=2$ appeared to be a relevant choice.

After this pruning step, the surviving tracks can be merged before updating to avoid redundant computation.

\section{B. Track merging}

Redundant tracks (i.e. tracks that correspond to the same object) may be generated and could imply unnecessary computation of state estimates. A popular approach is presented in [37]. It relies on assessing the similarity between the states of two tracks using the state vector estimates and covariance matrices: a criterion is defined on the statistical distance (i.e. Mahalanobis) or on each component, the choice of the criterion depending on the context. If the merging criteria meet, then the track of maximum likelihood is kept whereas the other are deleted. This technique has been implemented and is used in simulation.

In this study, merging criteria regarding the number of common observations, the lengths of tracks and the statistical distance between two tracks are used.

\section{Relevancy of the deletion steps}

The combinatorial complexity involved in tracking the LEO population is limited by the use of a gating function of sufficient precision and a score-function providing sufficient separability between the correct and incorrect tracks. Unlike the gating function, the score-function relies on a Multi Target Tracking (MTT) algorithm to delete tracks. Therefore, analyzing the behavior of the score (likelihood ratio) is of interest in order to implement a suitable MTT algorithm. This analysis shows that the log-likelihood ratio should not be used to delete unconfirmed tracks.

Figure 7 shows the evolution of the scores of correct tracks against the number of observations they comprise. This simulation has been made using the Space-Track TLE catalog and a SGP propagator on a simulation of observations from the sensor described in Section II over three days.

Figure 7 shows that on the mean the scores stay about zero when a low number of observations is available for each track and increase significantly afterward. This

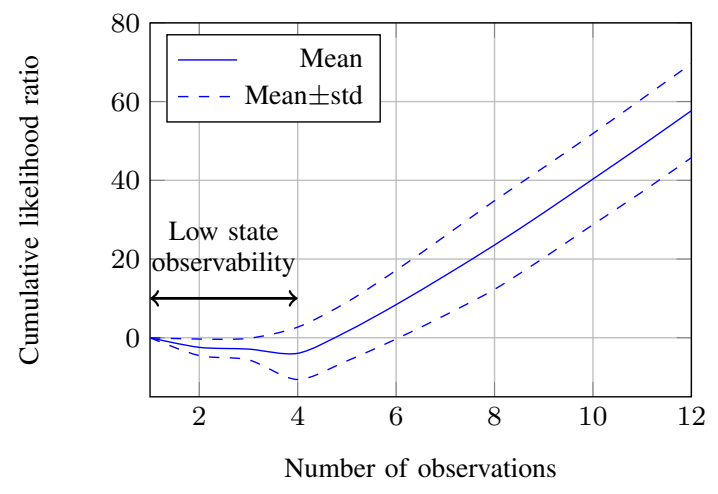

Figure 7. Evolution of the scores of correct tracks against the number of observations. A low state observability prevents the scores from increasing when the number of available observations is low.

behavior reflects that the likelihood is a poor criterion for pruning when the system observability is low whereas it is a good criterion when the system is observable. Therefore, the system observability should be taken into account when pruning. In the implemented algorithm, the likelihood is taken into account only for the tracks that enable a GaussNewton Least-Square (GNLS) convergence. Besides, any track sharing an observation with a confirmed track is deleted (this would be a consequence of a high-level pruning process) as well as any track containing more than 4 observations (see Figure 7) for which the GNLS failed to converge.

\section{Performance}

\section{A. Setting of the algorithm}

The implementation of the TOMHT involves many parameters which should be set to proper values. Most of them, such as the probabilities of type I and type II errors $\alpha$ and $\beta$ related to the thresholds defined by the SPRT [34] may be easily set according to the user requirements and to the peculiarities of the problem. However, some parameters involved in the new techniques presented in this work have a significant impact on the performance of the overall algorithm and require arduous tuning. These are:

- the threshold $\gamma_{\text {merging }}$ related to the statistical distance computed to assess the similarity between two tracks during the merging step (see Section IV-B),

- the coefficients of the diagonal matrix $\mathbf{D}_{\mathbf{X X}, 4}=$ $\operatorname{diag}\left(\kappa_{1}, \kappa_{2}, \kappa_{3}, \kappa_{4}\right)$ reflecting the uncertainty induced by the assumption of circular orbits made in III-C1.

Table II lists the main parameter values retained for the simulations. 
Table II

MAIN PARAMETER VALUES RETAINED FOR SIMULATION.

\begin{tabular}{ll}
\hline Parameter & Value \\
\hline \hline$\alpha$ & $1.157 \times 10^{-6}$ \\
$\beta$ & $10^{-3}$ \\
$\gamma_{\text {merging }}($ associated to $n)$ & $95 \%$ \\
$\kappa_{1}($ associated to $i)$ & $8 \times 10^{-9} \mathrm{rad} / \mathrm{s}$ \\
$\kappa_{2}($ associated to $\Omega)$ & $8 \times 10^{-3} \mathrm{rad}$ \\
$\kappa_{3}($ rad \\
$\kappa_{4}($ associated to $M)$ & $8 \times 10^{-9} \mathrm{rad}$ \\
\hline
\end{tabular}

The value of $\alpha$ is obtained from the SPRT assuming that a false confirmed track occurring once on 10 days on the mean is acceptable, and the values of $\beta$ and $\gamma_{\text {merging }}$ are arbitrarily chosen. The $\kappa$ 's are chosen from trajectories computed from the Space-Track catalog. Their values are adjusted so that the correlations of interest occur.

\section{B. Simulation conditions}

To assess the performance of the method proposed in this paper, the implementation of the TOMHT for tracking small LEO objects is applied on a set of simulated observations generated by 500 objects of the Space-Track catalog. The objects are chosen in the Space-Track catalog so that their orbits are close to generate a difficult tracking problem (the chosen objects all pass in the Field Of Regard (FOR) on a short time span). The propagation function $f_{\text {SGP }}$ provided in [24] (see Section II-B) is used to simulate the positions of the selected objects over 6 days. Then, the detections and associated measurements are simulated in the sensor-related assumptions provided in Section II-A.

No maneuver of the satellites has been simulated. Indeed, we assume that the LEO objects considered in this study are too small (less than $10 \mathrm{~cm}$ ) to carry fuel. The bigger objects such as maneuvering satellites can be cataloged by existing systems such as some components of the US SSN as well as the French system GRAVES.

False Alarms (FA) are randomly added to each scan where actual objects appear: 232,850 FAs are added to the 23,285 scans resulting from the simulation (i.e. $1 \mathrm{FA} / \mathrm{s}$ according to Section II), making the detection density realistic while avoiding the processing of clutteronly scans.

The resulting simulated observations are set as input of the implemented TOMHT based on the track initiation function proposed in this paper.

\section{Criteria of performance}

In order to assess the number of objects the algorithm would be able to track as well as its ability to delete tracks efficiently, we calculate the evolutions in time of three key performance criteria: the ratio of the number of objects the algorithm can track with no redundancy and a high degree of certainty (non-redundant, confirmed tracks) over the number of simulated objects (Figure 8 ); the total number of nodes (each node corresponds to an observation-to-track association hypothesis, the hypotheses being efficiently stored in a hierarchical tree structure) stored in memory (Figure 9); the ratio of the number of redundant confirmed tracks over the total number of confirmed tracks (Figure 10).

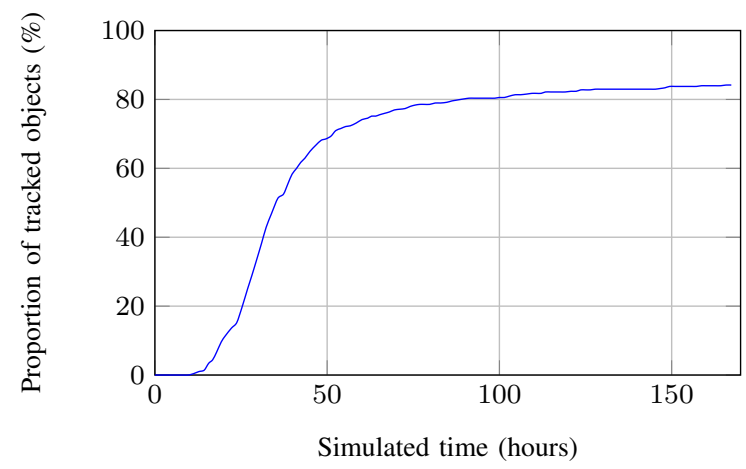

Figure 8. Evolution of the ratio of tracked objects over all the simulated objects.

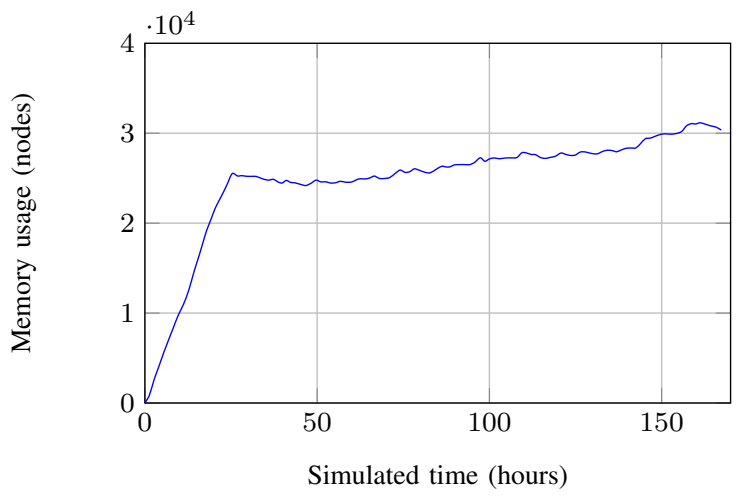

Figure 9. Evolution of the total number of nodes stored in memory.

At the end of the simulation, more than $84.4 \%$ of the simulated objects are tracked with no redundancy. The used memory stops increasing and less than $1 \%$ of the 


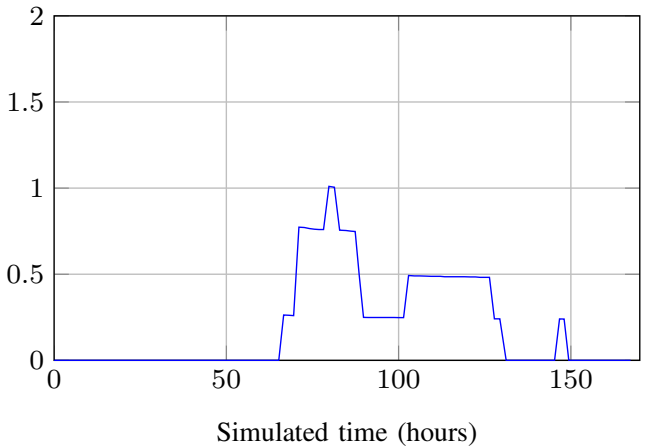

Figure 10. Evolution of the ratio of the number of redundant confirmed tracks over the total number of confirmed tracks.

total number of confirmed tracks are redundant during the simulated time.

\section{Discussion and leads for improvement}

The evolutions of the assessed criteria appear quite satisfactory. Indeed, the amount of used memory stagnates (Figure 9) and the redundant tracks are properly merged (Figure 10) while the ratio of tracked objects increases quickly after a short simulated time until it reaches a fair value of $84.4 \%$ (Figure 8 ).

The simulated objects are taken from the Space-Track catalog meaning that tracking $100 \%$ of the simulated objects corresponds to the tracking performance of the U.S. Space Surveillance Network (US SSN), which consists in several sensors distributed all over the world. In this study, a sensor of reduced FOR providing sparse observations with a rather low accuracy is assumed and still, the proposed algorithm could track successfully about $84.4 \%$ of the simulated objects. However, the proposed approach may result in a less reactive system compared to the US SSN as a few days are necessary to lock onto most of the objects but, as mentioned earlier, a set of non-maneuvering objects such as the space debris is assumed, making the system reactivity of lesser concern.

As for the amount of used memory (Figure 9), a clear stagnation occurs after about 24 hours of simulated time. This corresponds to the time required to accumulate enough observations for the first tracks to be confirmed or pruned. The slow increase of the amount of used memory after 24 hours is due to the prolongation of the confirmed tracks.

The ratio of tracked objects being an end point criterion (the system aims at tracking as many objects as possible), it is important to understand why it does not reach a higher value. This may be explained by two reasons: firstly, some objects may be lost; secondly, some objects with particular orbital states would not be tracked. However, no tracked objects were lost in simulation.

An object may never be tracked because: the object does not pass in the FOR at one revolution of interval; the object passes in the FOR at one revolution of interval but the pair of SAs it generates is rejected; the circular orbit assumed for a pair of SAs originated from the object does not correlate with the correct third SA.

In [12], simulations over a period of 30 days show that $99.8 \%$ of the LEO objects contained in the SpaceTrack TLE catalog pass in the FOR at one revolution of interval at least once and that $37.4 \%$ of the SAs from the same object are observed at one revolution of interval on the mean. As a consequence, the first reason mentioned to explain why an object may not be tracked should be discarded. However, the boundary and smoothing applied on the distributions resulting from the method proposed in [12] might result in too high a rejection rate.

As for the third reason, most of the objects are indeed of very low eccentricities i.e. more than $80 \%$ of the LEO objects contained in the Space-Track catalog have an eccentricity smaller than 0.02 , making quite relevant the circular orbit assumption for the IOD. However, the set of the objects that could not be tracked should be studied in order to determine whether or not specific orbit parameter values are more frequent comparing to the set of the tracked LEO objects.

\section{CONCLUSION}

The high combinatorial situation resulting from the low state observability of each SA has been avoided in the cataloging of Low-Earth-Orbit objects in a complex, realistic scenario comprising several hundreds of objects as well as false alarms. The tested implementation of the proposed approach, which relies on the statistical modeling of pairs of observations and a fast, circular orbit estimation, enables to track a large majority (84.4\%) of the objects simulated from the Space-Track catalog with a small amount of redundancy after convergence and a controlled memory usage. Therefore, the performance and its analysis tend to support the feasibility of the proposed approach.

From a systems design perspective, this approach appears to be promising taking into account that only one low-precision, narrow fence-type sensor enables a coverage of $84.4 \%$ whereas the system issuing the SpaceTrack catalog, which corresponds to the reference coverage 
of $100 \%$ in simulation, is composed of several sensors distributed all over the world.

In future works, the combinatorial complexity will be modeled in order to predict the computational requirements to solve a scenario with a realistic number of objects (i.e. more than 100,000).

\section{APPENDIX}

\section{A. Observation prediction using the Unscented Transform}

Let $\mathbf{X}_{t_{0}}$ and $\mathbf{P}_{\mathbf{X X}, t_{0}}$ be the state vector (orbital state) and the associated covariance matrix of dimension $n$ and $n \times n(n=6$ or $n=7)$. The statistical moments of this distribution is captured by $2 n+1$ sigma-points defined as:

$$
\begin{aligned}
& \chi_{t_{0}}^{0}=\mathbf{X}_{t_{0}} \\
& \chi_{t_{0}}^{i}=\mathbf{X}_{t_{0}}+\sqrt{(n+\kappa) \mathbf{P}_{\mathbf{X X}} \mathbf{X}_{t_{0}}} \\
& \chi_{t_{0}}^{i+n}=\mathbf{X}_{t_{0}}-\sqrt{(n+\kappa) \mathbf{P}_{\mathbf{X X}} \mathbf{X}_{, t_{0}}}
\end{aligned}
$$

where $\kappa$ is a scaling parameter and $\sqrt{\mathbf{P}_{\mathbf{X X}}{ }_{, t_{0}}^{i}}$ is the $i$-th column of an L-triangular matrix obtained from a Cholesky decomposition of $\mathbf{P}_{\mathbf{X X}, t_{0}}$.

The $\sigma$-points are propagated through $f_{\text {SGP }}$ and $h$ ( $h$ denotes the mapping from ECF to ENU) at time $t$ :

$$
\begin{aligned}
\overline{\mathbf{z}}_{t}= & \sum_{i=0}^{2 n} \varphi^{i}\left(h \circ f_{\mathrm{SGP}}\right)\left(\chi_{t_{0}}^{i}, t\right) \\
\mathbf{P}_{\overline{\mathbf{z}} \overline{\mathbf{z}}, t}= & \sum_{i=0}^{2 n} \varphi^{i}\left(\left(h \circ f_{\mathrm{SGP}}\right)\left(\chi_{t_{0}}^{i}, t\right)-\overline{\mathbf{z}}_{t}\right) \\
& \times\left(\left(h \circ f_{\mathrm{SGP}}\right)\left(\chi_{t_{0}}^{i}, t\right)-\overline{\mathbf{z}}_{t}\right)^{\mathrm{T}}
\end{aligned}
$$

where

$$
\begin{array}{lll}
\varphi^{0} & =\frac{\kappa}{n+\kappa} \\
\varphi^{i} & =\frac{1}{2(n+\kappa)} \\
\varphi^{i+n} & =\frac{1}{2(n+\kappa)}
\end{array}
$$

Then, $\overline{\mathbf{z}}_{t}$ and $\mathbf{P}_{\overline{\mathbf{z}} \overline{\mathbf{z}}, t}$ can be used to correlate new observations at time $t$ with the track of state $\mathbf{X}_{t_{0}}$.

\section{B. Principle of the One-Class Support Vector Machine}

The One-Class Support Vector Machine (OC-SVM) is an extension of the better-known bi-class SVM [29][30][31] that aims at defining a simple decision function $f_{\text {OC-SVM }}$ in order to label input vectors $\{\mathbf{x}\}$ as belonging or not belonging to a given class $H_{1}$. To this purpose, the OC-SVM requires a set of "labeled data" $\left\{\mathbf{x}_{H_{1}}\right\}$ and considers the origin as the prototype of a second class. Since a separating hyperplan is not optimal or may not even exist in the original space $E$, a mapping function $\Phi$ is introduced:

$$
\Phi: \begin{aligned}
& E \longrightarrow F \\
& \mathbf{x} \longrightarrow \Phi(\mathbf{x})
\end{aligned}
$$

where $F$ is a feature space in which a separating hyperplan $S_{F}$ exists. In $F$, the separating hyperplan $S_{F}$ can be defined by the couple $\{\mathbf{w}, b\} \in F \times \mathbb{R}$ :

$$
\mathbf{x} \in S_{F} \Leftrightarrow \mathbf{w} \cdot \mathbf{x}-b=0
$$

The distance of $S_{F}$ to the origin is $\frac{b}{\|\mathbf{w}\|}$ and can be maximized solving the following quadratic program:

$$
\begin{array}{r}
\underset{\mathbf{w}, b, \boldsymbol{\xi}}{\arg \min } \frac{1}{2}\|\mathbf{w}\|^{2}-b+\frac{1}{\nu l} \sum_{i} \xi_{i} \\
\text { subject to } \mathbf{w} \cdot \Phi\left(\mathbf{x}_{H_{1}, i}\right) \geq b-\xi_{i}, \xi_{i}>0
\end{array}
$$

where $\nu$ is the regularization parameter introduced in Eq. (6), $l$ the number of training samples $\mathbf{x}_{H_{1}, i}$ and $\boldsymbol{\xi}$ a vector of slack variables which enable to deal with mislabelled data and regularize the classification. Since nonzero slack variables are penalized in the objective function, the function $f_{\mathrm{OC}-\mathrm{SVM}}$ defined as:

$$
f_{\mathrm{OC}-\mathrm{SVM}}(\mathbf{x})=\operatorname{sgn}(\mathbf{w} \cdot \Phi(\mathbf{x})-b)
$$

is expected to be positive for most of the training samples $\mathbf{x}_{H_{1}, i}$ and therefore in the corresponding half-space of $F$. Back in $E$, this half-space corresponds to the minimal volume $C$ that encompasses at least a fraction $1-\nu$ of the underlying probability distribution of the set $\left\{\mathbf{x}_{H_{1}}\right\}$, defined as the $(1-\nu)$-quantile $Q(1-\nu)$ in Eq. (6).

The "kernel trick" enables to express $f_{\text {OC-SvM }}$ in a more straightforward way. Using the method of Lagrange multipliers, w can be expressed as:

$$
\mathbf{w}=\sum_{i} \alpha_{i} \Phi\left(\mathbf{x}_{H_{1}, i}\right)
$$

where the $\alpha_{i}$ are the Lagrange multipliers. Then, $f_{\text {OC-SVM }}$ can be rewritten as:

$$
\begin{aligned}
f_{\mathrm{OC}-\mathrm{SVM}}(\mathbf{x}) & =\operatorname{sgn}\left(\sum_{i} \alpha_{i} \Phi\left(\mathbf{x}_{H_{1}, i}\right) \cdot \Phi(\mathbf{x})-b\right) \\
& =\operatorname{sgn}\left(\sum_{i} \alpha_{i} k\left(\mathbf{x}_{H_{1}, i}, \mathbf{x}\right)-b\right)
\end{aligned}
$$

where $k$ is a kernel function (such as a Gaussian kernel) defined so that $k\left(\mathbf{x}_{H_{1}, i}, \mathbf{x}\right)=\Phi\left(\mathbf{x}_{H_{1}, i}\right) \cdot \Phi(\mathbf{x})$ in order to avoid an explicit determination of $\Phi$. 


\section{REFERENCES}

[1] O. of Science en Technological Policy, "Interagency report on orbital debris," in The National Science and Technology Council, Commitee on Transportation Research and Development, 1995.

[2] Pate-Cornell, E. and Sachon, M., "Risks of particle hits during space walks in low earth orbit," Aerospace and Electronic Systems, IEEE Transactions on, 2001.

[3] Hody, E., Bertrand, S., Muller, F., Brudieu, P. and Alby, F., "Analysis of the residual risk of lethal collisions for LEO satellites due to non catalogued objects," 62nd International Astronautical Congress, Cape Town, South Africa, 2011.

[4] DeMars, K. J., Jah, M. K. and Schumacher, P. W., "Initial orbit determination using short-arc angle and angle rate data," Aerospace and Electronic Systems, IEEE Transactions on, 2012.

[5] Schumacher, P. W. Jr., "US naval space surveillance upgrade program 1999-2003," in 7th US/Russian Space Surveillance Workshop Proceedings, Naval Postgraduate School, CA, 2007.

[6] Michal, T., Eglizeaud, J. P. and Bouchard, J., "GRAVES: the New French System for Space Surveillance," in Proceedings of the 4th European Conference on Space Debris (ESA SP-587), 2005.

[7] Hoots, F. R. and Roehrich, R. L., "Models for propagation of NORAD element sets, SpaceTrack report no. 3," tech. rep., US Air Force, 1980

[8] Kessler, D., "Collisional cascading: The limits of population growth in low earth orbit," Advances in Space Research, 1991

[9] Stansbery, E. G., "Growth in the number of SSN tracked orbital objects," 55th International Astronautical Congress, Vancouver, Canada, 2004.

[10] Knott, E. F., Shaeffer, J. F. and Tuley, M. T., Radar Cross Section. Artech House Radar Library, 1985.

[11] Stokely, C. L., Foster J. L. Jr., Stansbery, E. G., Benbrook, J. R. and Juarez, Q., "Haystack and HAX radar measurements of the orbital debris environment; 2003," tech. rep., NASA Lyndon B. Johnson Space Center, 2006.

[12] Castaings, T., Pannetier, B., Muller, F. and Rombaut, M., "Sparse data association for low earth orbit tracking," in Aerospace Conference, IEEE, 2012.

[13] Castaings, T., Muller, F., Pannetier, B. and Rombaut, M., "Track initiation using sparse radar data for low earth orbit objects," in 63rd International Astronautical Congress, Naples, Italy, 2012.

[14] Blackman, S. S. and Popoli, R., Design and Analysis of Modern Tracking Systems. Artech House Radar Library, 1999.

[15] Kurien, T., Issues in the Design of Practical Multitarget Tracking Algorithms. Ed. Y. Bar-Shalom, Artech House, 1990.

[16] Bar-Shalom, Y., Multitarget-Multisensor Tracking: Principle and Techniques. Burlington, MA: Academic Press, 1995.

[17] Blackman, S. S., "Multiple hypothesis tracking for multiple target tracking," Aerospace and Electronic Systems Magazine, IEEE, vol. 19, no. 1, pp. 5-18, 2004.

[18] Chang, K. C., Mori, S. and Chong, C. Y., "Evaluating a multiplehypothesis multitarget tracking algorithm," Aerospace and Electronic Systems, IEEE Transactions on, 1994.

[19] Sinha, A., Ding, Z., Kirubarajan, T. and Farooq, M., "Track quality based multitarget tracking approach for global nearest-neighbor association," Aerospace and Electronic Systems, IEEE Transactions on, 2012.

[20] Vallado, D. A., Fundamentals of Astrodynamics and Applications. Microcosm Press, 4940 West 147th Street, Hawthorne, CA 902506708 USA and Springer, 233 Spring Street, New York, NY 10013 USA, 2007.

[21] Shen, H. and Tsiotras, P., "Using Battin's method to obtain multiplerevolution Lambert's solutions," in Paper AAS-03-568 presented at the AAS/AIAA Astrodynamics Specialist Conference. Big Sky, MT., 2003.

[22] Raol, J. R. and Sinha, N. K., "On the orbit determination problem," Aerospace and Electronic Systems, IEEE Transactions on, 1985.
[23] Barrar, R. B. and Deutsch, R., "Determination of satellite trajectories from track-while-scan radar measurements," Military Electronics, IRE Transactions on, 1961.

[24] Vallado, D. A., Crawford, P., Hujsak, R. and Kelso, T. S., "Revisiting SpaceTrack report no. 3," tech. rep., American Institute of Aeronautics and Astronautics, 2006.

[25] Deirmendjian, D., "Electromagnetic scattering on spherical polydispersions," tech. rep., RAND Corporation, 1969.

[26] Lane, M. H., Fitzpatrick, P. M. and Murphy, J. J., "On the representation of air density in satellite deceleration equations by power functions with integral exponents," tech. rep., Air Force Systems Command, 1962.

[27] Baase, S., Computer Algorithms: Introduction to Design and Analysis. Reading, MA: Addison-Wesley, 1978.

[28] Schölkopf, B., Platt, J. C., Shawe-Taylor, J., Smola, A. J. and Williamson, R. C., "Estimating the support of a high-dimensional distribution," Neural Computation, 2001.

[29] Vapnik, V. N., The nature of statistical learning theory. New York: Springer-Verlag, 1995.

[30] Fauvel, M., Tarabalka, Y., Benediktsson, J. A. and Chanussot, J., "Advances in spectral-spatial classification of hyperspectral images," Proceedings of the IEEE, 2013.

[31] Le Saux, B. and Boujemaa, N., "Image database clustering with SVM-based class personalization," Proceedings of SPIE, 2004.

[32] Parzen, E., "On estimation of a probability density function and mode," Annals of Mathematical Statistics, 1962.

[33] Bar-Shalom, Y., Blackman, S. S. and Fitzgerald, R. J., "Dimensionless score function for multiple hypothesis tracking," Aerospace and Electronic Systems, IEEE Transactions on, 2007.

[34] Wald, A., "Sequential tests of statistical hypotheses," Annals of Mathematical Statistics, 1945.

[35] Cao, Z. B., Xu, J., Ma, J. B., Hu, W. D. and Fang, A. L., "A research on the orbit determination method by means of sparse data of electronic fences," Chinese Astronomy and Astrophysics, 2009.

[36] Julier, S. J., Uhlmann, J. K. and Durrant-Whyte, H. F., "A new approach for filtering nonlinear systems," in Proceedings of the American Control Conference, Seattle, Washington, pages 16281632, 1995

[37] Blackman, S. S., Multiple Target Tracking with Radar Applications. Artech House, 1986.

Thibaut Castaings graduated with a $\mathrm{Ph}$. D. in signal processing from the University of Grenoble in 2014 and with an Engineer Degree from the Grenoble Institute of Technology in 2010. He is currently a research engineer at ONERA, the French Aerospace Lab, working mainly on space surveillance.

Benjamin Pannetier received his B.S. mathematical degree from the University of Marne la Vallée, and his Ph. D. in automatic control and signal processing from the University of Grenoble in 2006. Since 2005 , he is a research engineer at ONERA. His research interests include target tracking, detection/estimation theory and data fusion for battlefield surveillance systems for the French army. With Professor Rombaut (University of Grenoble), he is working on a new approach for the abnormal behaviour detection. 
Florent Muller graduated from Ecole Supérieure d'Electricité in 2001. He joined former Aerospatiale (now MBDA) in 2001, as a research engineer. He worked on mission planning algorithms, fire control algorithms and global performance evaluation of a missile. He entered ONERA in 2006 as a Deployable Early Warning Radar project manager. He also took part to different defence architecture system evaluation, as well as Space Situational Awareness System performances evaluation. $\mathrm{He}$ is now head of the Space and Defence Systems Unit in the System Design and Performance Evaluation Department (DCPS).

Michèle Rombaut graduated from the Ecole Universitaire d'Ingénieurs de Lille, France, and received her Ph. D. from the University of Lille in electronic systems. Since 1985, she has been an Associate Professor at the Université de Technologie de Compiègne and a Professor since 1994. Since 2002, she has been conducting her research at GIPSA-Lab, Université de Grenoble, mainly in data fusion and transferable belief model. 